\title{
Interactions between Ag nanoclusters on carburized W(110)
}

\author{
Martin Gabl, Magdalena Bachmann, Norbert Memmel, and Erminald Bertel \\ Institute of Physical Chemistry, University of Innsbruck Innrain 52a, A-6020 Innsbruck, Austria \\ (Received 17 December 2008; revised manuscript received 27 February 2009; published 27 April 2009)
}

\begin{abstract}
Silver nanoclusters arranged in quasi-one-dimensional chains with a nearest-neighbor cluster-cluster distance of $1.4 \mathrm{~nm}$ were prepared on the $R(15 \times 12)-\mathrm{C} / \mathrm{W}(110)$ surface. The silver cluster chains form local thermodynamic equilibrium structures. Interactions between neighboring clusters are addressed by investigating the length distributions of silver cluster chains with scanning tunneling microscopy. Comparison with theoretical expectations derived from a one-dimensional Ising model yields evidence for a slightly repulsive interaction energy of about $20-30 \mathrm{meV}$.
\end{abstract}

DOI: 10.1103/PhysRevB.79.153409

PACS number(s): 81.16.Dn

One of the attractive features of nanotechnology besides mere miniaturization is the possibility to exploit quantum size effects and to steer the properties of nanoscale structures by choosing the proper size and shape. However, interactions between nano-objects may also influence the properties of the nanostructures and thus alter the simple property size/ shape relationship. Quite often these interactions are neglected as the relevant distances are considered to be too large to allow appreciable modification of the energy balance. However, this assumption may break down in the case of tightly packed nanoparticles (i.e., with interparticle distances of only a few nanometers) on solid surfaces due to long-range substrate-mediated interactions. ${ }^{1-3}$ Substratemediated interactions between single adatoms have been studied quite extensively in the past. For example, it has been shown both experimentally ${ }^{4,5}$ and theoretically ${ }^{6}$ that interactions between single $\mathrm{Cu}$ adatoms on $\mathrm{Cu}(111)$ are as large as $1 \mathrm{meV}$ at distances of 1-2 $\mathrm{nm}$. For Ce adatoms on $\mathrm{Ag}(111)$ a similar interaction energy was derived at distances of $3 \mathrm{~nm} .{ }^{7}$ For Re atoms on W(110) interaction strengths of about $15 \mathrm{meV}$ were reported in the distance range $1-2 \mathrm{~nm} .{ }^{8}$ At low temperatures substrate-mediated interactions can be exploited to arrange adatoms into nanoscale superstructures such as two-dimensional hexagonal superlattices, ${ }^{7}$ triangular, ${ }^{9}$ or circular $^{10}$ arrangements or even onedimensional (1D) chains. ${ }^{11-13}$ In case of clusters/islands consisting of several atoms the interactions will be larger than for single adatoms. Nevertheless studies on cluster-cluster interactions are rare. ${ }^{14,15}$ A rough estimate of the strength of cluster-cluster interactions can be obtained from RudermannKittel-Kasuya-Yosida (RKKY) theory, which describes substrate-electron mediated interactions. According to this theory the interaction between two objects scales with the product of the perturbing strengths of the individual objects. Assuming that this strength scales linearly with the number of atoms at the rim of the cluster, ${ }^{16}$ for the interaction of two clusters with seven atoms each [six atoms at the rim and one in the center; see schematic in Fig. 1(a)], a roughly fortyfold increase in interaction energy appears possible. Such interaction energies would exceed the thermal energy $k_{B} T$ at room temperature $(25 \mathrm{meV})$ and hence can no longer be ignored. In a recent work interactions between $\mathrm{Br}$ islands on $\mathrm{Cu}(111)$ were studied and indeed an appreciable interaction strength of $\approx 25 \mathrm{meV}$ was reported for distances around $2 \mathrm{~nm} .{ }^{14}$

In the experiments cited above particle-particle interac- tions were derived from pair correlations or radial distribution functions, respectively, by evaluating the potential of mean force, which in dilute systems (i.e., at low densities) approximates the pair-interaction potential. ${ }^{17}$ In the present study we use an alternative approach. It is based on the (onedimensional) Ising model, which can be used to describe interacting objects distributed on a regular grid. We provide evidence that in the present system, which consists of $\mathrm{Ag}$ nanoclusters arranged in one-dimensional chains on a carburized W(110) surface, weak repulsive interactions exist between neighboring clusters (distance $1.4 \mathrm{~nm}$ ), with a strength of around $20-30 \mathrm{meV}$.

Experiments were carried out in an UHV system (base pressure of $1 \cdot 10^{-10} \mathrm{mbar}$ ), equipped with a DME (Danish Micro Engineering) room-temperature scanning-tunneling microscope $(\mathrm{STM})$. The $R(15 \times 12) \mathrm{C}-\mathrm{W}(110)$ template was prepared by thermolysis of ethene at a pressure of $5 \cdot 10^{-8}$ mbar and temperatures at $1250-1700 \mathrm{~K}$, followed by a flash to $2300 \mathrm{~K}$ and rapid cooling $(\approx 70 \mathrm{~K} / \mathrm{s})$. Ag was deposited from a resistively heated alumina crucible with deposition rates between 0.03 and $0.3 \mathrm{ML} / \mathrm{min}$, where 1 monolayer (ML) refers to the atom density of the $\mathrm{W}(110)$ surface, i.e., $1.41 \cdot 10^{15}$ atoms $/ \mathrm{cm}^{2}$.

The $R(15 \times 12)-\mathrm{C} / \mathrm{W}(110)$ surface has a large rectangular unit cell of $1.37 \times 3.10 \mathrm{~nm}$, equivalent to the area of $60 \mathrm{~W}$ atoms in an unreconstructed W(110) surface. ${ }^{18}$ The unit-cell axes are oriented along the $[1 \overline{1} 1]$ and [ $\overline{1} 12]$ directions, respectively. Due to the mirror symmetry of the W(110) surface two equivalent domains exist. Each unit cell of the C/W template provides a possible adsorption site for a metal cluster. ${ }^{19}$ Thus - due to the anisotropic size of the unit cellquasi-one-dimensional chains of clusters can be grown. A representative example for deposition of silver clusters in the temperature range of $350-600 \mathrm{~K}$ is shown in Fig. 1(a). As is evident from this figure, the clusters exhibit a narrow size distribution. Close inspection of the nanoclusters revealed a hexagonal shape, suggesting a preference for seven-atom clusters. ${ }^{19}$ A tentative cluster model is indicated in Fig. 1(a). As discussed in Ref. 19, this strong size preference is mainly determined by the interaction with the substrate, i.e., by the number of strong adsorption sites within each unit cell. At the "ideal" coverage of $0.12 \mathrm{ML}$ virtually all unit cells are occupied by such Ag heptamers.

We assign these heptamer clusters to thermodynamic 

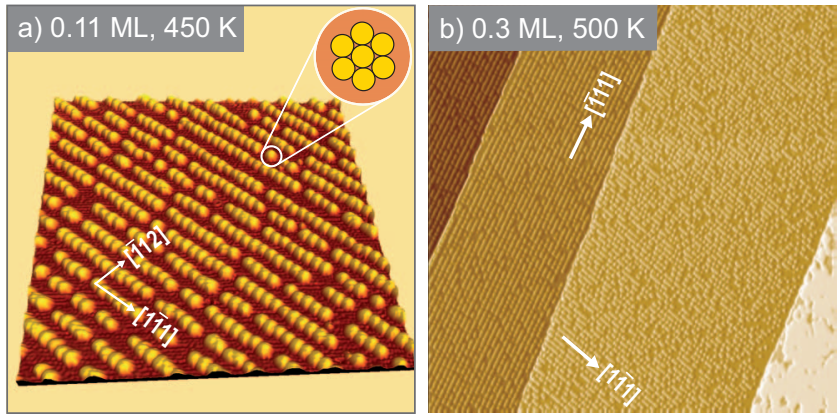

FIG. 1. (Color) (a) STM image of self-assembled Ag clusters deposited at $450 \mathrm{~K}$ on $R(15 \times 12)-\mathrm{C} / \mathrm{W}(110)$, with image size of $47 \times 47 \mathrm{~nm}$. Inset: tentative cluster model. (b) $0.3 \mathrm{ML} \mathrm{Ag} \mathrm{deposited}$ at $T \approx 500 \mathrm{~K}$, with image size of $250 \times 250 \mathrm{~nm}$. Cluster arrays are formed, although the global coverage $(0.3 \mathrm{ML})$ exceeds the coverage of $0.12 \mathrm{ML}$ which can be accommodated in the cluster array. Due to the high deposition temperature excess Ag atoms are transported to clean tungsten terraces where they agglomerate in large mono- and double-layer islands (lower right corner of image).

equilibrium structures for several reasons: first, clusters are observed in a wide temperature range (350-600 K). Second, for coverages below the ideal coverage, the average cluster size is constant. Thus the cluster size is not determined by kinetics or limited supply of Ag atoms. Third, Ag heptamers are formed even at coverages in excess of the ideal $0.12 \mathrm{ML}$, provided that the temperature is sufficiently high ( $T$ $\approx 500 \mathrm{~K}$ ) for the Ag atoms to be mobile. Under these conditions excess $\mathrm{Ag}$ atoms (i.e., Ag atoms beyond 0.12 ML) diffuse away and agglomerate on clean W(110) patches (which are always present on the surface), while the Ag heptamers remain on carburized $(15 \times 12)$ terraces. In the lower right corner of Fig. 1(b) such a clean W(110) patch covered with a dense Ag film can be seen.

Having obtained convincing evidence that the Ag clusters are thermodynamically stable entities on (15 $\times 12)-\mathrm{C} / \mathrm{W}(110)$ terraces, we are now in the position to address the question of cluster-cluster interactions by analysis of the positions of missing clusters ("vacancies") for suboptimal local coverages $(\theta<0.12 \mathrm{ML})$. In the absence of cluster-cluster interactions vacancies should be randomly distributed on the terraces. In contrast, for attractive clustercluster interactions longer, noninterrupted chains of both clusters and vacancies are expected. For repulsive interactions the opposite effect will take place. In the noninteracting case the cluster and vacancy chain-length distributions are easily obtained analytically. ${ }^{20}$ If $p$ and $1-p$ denote the probabilities that a $(15 \times 12)$ cell is covered with a cluster or vacancy, respectively, the probability $p_{s}$ for a cluster chain of length $s$ (terminated by a vacancy on each side) is given as

$$
p_{s}=p^{s}(1-p)^{2} \text {. }
$$

For vacancy chains an analogous formula holds, with $p$ and (1- $p$ ) interchanged. In order to quantitatively asses the effect of eventual interactions, we assume that the binding energy of each cluster can be split into a cluster-substrate interaction $\left(E_{z}\right)$ and a cluster-cluster interaction $\left(E_{x}\right)$, i.e., $E_{\text {bind }}=E_{z}$ $+n^{*} E_{x}$, where $n=0,1,2$ is the number of nearest-neighbor clusters. In this approximation the problem is reduced to that of the one-dimensional Ising model with nearest-neighbor interactions only. The occupation number of a template unit cell, i.e., 0 or 1 , takes the role of the pseudospin in the system. Since for a fixed number of clusters the total binding strength to the substrate is independent of the cluster arrangement, the chain-length distribution in thermal equilibrium depends only on the ratio $\varepsilon=E_{x} / k T$ of the lateral interaction energy $E_{x}$ to the thermal energy $k T$ ( $k$ denotes the Boltzmann constant). For the chain-length distribution in the 1D-Ising model the following analytical expression was derived recently, ${ }^{21}$

$$
p_{s}=\frac{\alpha^{2}}{p}\left(1-\frac{\alpha}{p}\right)^{s-1} \quad \text { with } \quad \alpha=\frac{\sqrt{1+4 p(1-p)\left(e^{\varepsilon}-1\right)}-1}{2\left(e^{\varepsilon}-1\right)} .
$$

Thus in a semilog plot $\left[\log \left(p_{s}\right)\right.$ vs $\left.s\right]$ a linear behavior is predicted. In the limit of zero interaction $(\varepsilon \rightarrow 0)$ Eq. (2) reduces to Eq. (1).

To derive the interaction strengths $\varepsilon$ or $E_{x}$, respectively, Ag cluster chains were prepared at different deposition temperatures and with different "filling factors" $p$. Then cluster
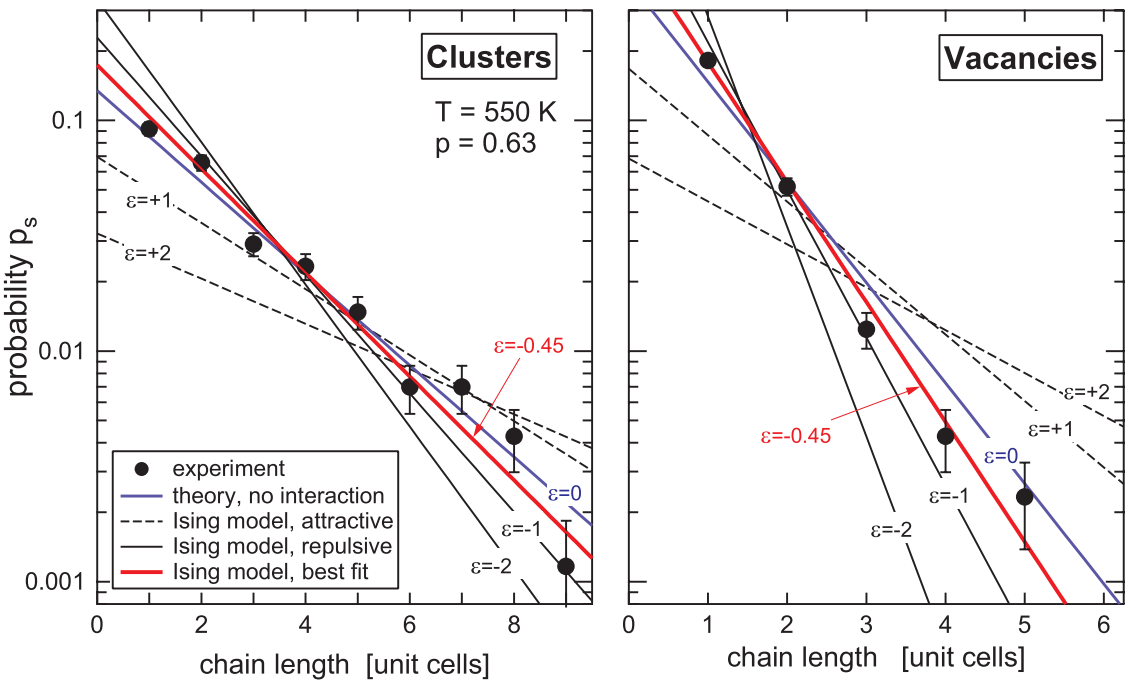

$153409-2$
FIG. 2. (Color) Semilog plot of Ag cluster and vacancy chainlength distributions on $R(15$ $\times 12)-\mathrm{C} / \mathrm{W}(110)$ with $p=63 \%$ of the $(15 \times 12)$ unit cells occupied with clusters. Symbols denote experimental data points. Lines denote theoretical calculations based on a 1D-Ising model for various cluster-cluster interaction parameters $\varepsilon=E_{x} / k T$. Attractive (repulsive) interactions are marked by dashed (solid) lines. The blue line indicates the interaction-free case; the red line the best fit. 
TABLE I. Interaction parameters $\varepsilon\left(=E_{x} / k T\right)$ and interaction energies $\left(E_{x}\right)$ derived from cluster and vacancy chain-length distributions for experiments at various deposition temperatures $T$ and filling factors $p$. The filling factor $p$ denotes the fraction of unit cells occupied with silver clusters.

\begin{tabular}{cccc}
\hline \hline$T$ & & & $E_{x}$ \\
$(\mathrm{~K})$ & $p$ & $\varepsilon$ & $(\mathrm{meV})$ \\
\hline 350 & 0,47 & $-1,12$ & -34 \\
400 & 0,64 & $-0,60$ & -21 \\
400 & 0,35 & $-1,45$ & -50 \\
450 & 0,83 & $-0,11$ & -4 \\
500 & 0,78 & $-0,34$ & -15 \\
550 & 0,63 & $-0,45$ & -21 \\
& & average & $-24 \pm 7$ \\
\hline \hline
\end{tabular}

and vacancy chain-length distributions as derived from STM images were compared to the theoretical expectations according to Eqs. (1) and (2). Figure 2 depicts a representative example for an experiment carried out with a filling factor $p=0.63$ at a deposition temperature of $550 \mathrm{~K}$ (symbols). For comparison, calculated length distributions resulting from random positioning of vacancies $(\varepsilon=0$, blue color) as well as for various interaction strengths $\varepsilon$ are shown as solid and dashed lines. As expected, theoretical cluster and vacancy distributions become broader for attractive interactions $(\varepsilon$ $>0$, dashed lines), while for repulsive interactions $(\varepsilon<0$, solid lines) smaller chain lengths are preferred. Experimental data points are fairly close to the interaction-free case; however closer inspection reveals that they are better described by a slightly repulsive interaction. Fitting the experimental data by Eq. (2) yields $\varepsilon=-0.32$ and $\varepsilon=-0.55$, respectively, if cluster and vacancy chain-length distributions are fitted individually. Fitting both curves simultaneously yields $\varepsilon=-0.45$ (red line in Fig. 2), corresponding to an interaction energy $E_{x}=-21 \mathrm{meV}$ at $T=550 \mathrm{~K}$. The interaction energies $E_{x}$ derived in this way for various experiments are listed in Table I. The energy values show some scatter; however in all cases interaction energies are found to be small $(<50 \mathrm{meV})$ and negative. The mean value amounts to $-24 \mathrm{meV}$ with a standard error of $\pm 7 \mathrm{meV}$. Thus our data provide evidence for a weak repulsive interaction between neighboring clusters. The derived interaction strength of $20-30 \mathrm{meV}$ appears reasonable. It is in the same range as predicted by the simple upscaling argument for $\mathrm{Cu}$ heptamer clusters on $\mathrm{Cu}(111)$ discussed in Sec. I and it is similar to the interaction energy of $\mathrm{Br}$ clusters on $\mathrm{Cu}(111)$ for distances of $\approx 2 \mathrm{~nm}$. Since the cluster-cluster distance of $1.4 \mathrm{~nm}$ in the present system amounts to several atomic distances, these interactions have to be substrate mediated, either by elastic strain fields or by indirect electronic interactions. Elastic interactions decrease with $d^{-3}$ ( $d$ denotes the distance between interacting objects), ${ }^{2}$ whereas interactions mediated by two-dimensional electronic surface states decrease as $d^{-2}$ or even $d^{-1}$ in onedimensional systems. ${ }^{4-6,22,23}$ Thus electronic interactions will always dominate at larger distances. As shown in Ref. 24 for $\mathrm{Fe}$ adatoms on $\mathrm{Cu}(111)$ electronic interactions exceed elastic ones by more than an order of magnitude already at distances around $1.3 \mathrm{~nm}$. Thus we attribute the interactions found in the present system primarily to indirect electronic interactions. Note that bulk tungsten exhibits a Fermi surface with portions of rather flat, parallel faces normal to [111], i.e., with a quasi-one-dimensional Fermi-surface topology in the direction along the Ag cluster rows. Furthermore, electronic surface states and resonances have also been observed and predicted on clean W(110). ${ }^{25-28}$ Unfortunately, neither photoemission experiments nor theoretical calculations on the electronic structure of the carbon-modified $\mathrm{R}$ (15 $\times 12) \mathrm{C}-\mathrm{W}(110)$ template are currently available. Thus, pinning down the detailed interaction mechanism remains a task for the future.

In summary, we investigated the distribution of silver clusters and vacancies on the $(15 \times 12) \mathrm{C}-\mathrm{W}(110)$ template. The data present evidence for a slightly repulsive clustercluster interaction in the range of $20-30 \mathrm{meV}$. As expected the interaction between clusters is considerably stronger than between single adatoms at similar distances. Nevertheless the interaction energy is small and at room temperature or above yields only small deviations from the interaction-free situation. However, we conclude that in low-temperature experiments the possible influence of such interactions can in general not be neglected and should always be considered for tightly packed nanostructures on surfaces.

Financial support by the Austrian Science Fund (Grant No. S9004-N20) is gratefully acknowledged.
${ }^{1}$ J. Koutecky, Trans. Faraday Soc. 54, 1038 (1958).

${ }^{2}$ K. H. Lau and W. Kohn, Surf. Sci. 65, 607 (1977).

${ }^{3}$ K. H. Lau and W. Kohn, Surf. Sci. 75, 69 (1978).

${ }^{4}$ J. Repp, F. Moresco, G. Meyer, K. H. Rieder, P. Hyldgaard, and M. Persson, Phys. Rev. Lett. 85, 2981 (2000).

${ }^{5}$ N. Knorr, H. Brune, M. Epple, A. Hirstein, M. A. Schneider, and K. Kern, Phys. Rev. B 65, 115420 (2002).

${ }^{6}$ V. S. Stepanyuk, L. Niebergall, A. N. Baranov, W. Hergert, and P. Bruno, Comput. Mater. Sci. 35, 272 (2006).

${ }^{7}$ F. Silly, M. Pivetta, M. Ternes, F. Patthey, J. P. Pelz, and W. D. Schneider, Phys. Rev. Lett. 92, 016101 (2004).
${ }^{8}$ T. T. Tsong, Phys. Rev. Lett. 31, 1207 (1973).

${ }^{9}$ G. Manai, K. Radican, F. Delogu, and I. V. Shvets, Phys. Rev. Lett. 101, 165701 (2008).

${ }^{10}$ V. S. Stepanyuk, N. N. Negulyaev, L. Niebergall, R. C. Longo, and P. Bruno, Phys. Rev. Lett. 97, 186403 (2006).

${ }^{11}$ H. F. Ding, V. S. Stepanyuk, P. A. Ignatiev, N. N. Negulyaev, L. Niebergall, M. Wasniowska, C. L. Gao, P. Bruno, and J. Kirschner, Phys. Rev. B 76, 033409 (2007).

${ }^{12}$ A. Schiffrin, J. Reichert, W. Auwarter, G. Jahnz, Y. Pennec, A. Weber-Bargioni, V. S. Stepanyuk, L. Niebergall, P. Bruno, and J. V. Barth, Phys. Rev. B 78, 035424 (2008). 
${ }^{13}$ C. Liu, T. Uchihashi, and T. Nakayama, Phys. Rev. Lett. 101, 146104 (2008).

${ }^{14}$ S. U. Nanayakkara, E. C. Sykes, L. C. Fernandez-Torres, M. M. Blake, and P. S. Weiss, Phys. Rev. Lett. 98, 206108 (2007).

${ }^{15}$ R. C. Longo, V. S. Stepanyuk, and J. Kirschner, J. Phys.: Condens. Matter 18, 9143 (2006).

${ }^{16}$ This scaling is suggested by the work on $\mathrm{Br}$ islands on $\mathrm{Cu}(110)$ [Ref. 14], which shows that the substrate electrons mediating the interaction are mainly scattered by atoms at island rim. For extremely small clusters such as the heptamers depicted in Fig. 1(a) basically all cluster atoms reside at the cluster rim.

${ }^{17}$ T. L. Hill, Statistical Mechanics (McGraw-Hill, New York, 1956); S. A. Rice and P. Gray, The Statistical Mechanics of Simple Liquids (Interscience, New York, 1965).

${ }^{18}$ R. Baudoing and R. M. Stern, Surf. Sci. 10, 392 (1968).

${ }^{19}$ M. Bachmann, M. Gabl, C. Deisl, N. Memmel, and E. Bertel, Phys. Rev. B 78, 235410 (2008).

${ }^{20}$ D. Stauffer and A. Aharony, Perkolationstheorie (VCH, Weinheim 1995), p. 19ff.
${ }^{21}$ M. B. Yilmaz and F. M. Zimmermann, Phys. Rev. E 71, 026127 (2005).

${ }^{22}$ T. L. Einstein, in Physical Structure of Solid Surfaces, edited by W. N. Unertl, Handbook of Surface Science Vol. 1, edited by S. Holloway and N. V. Richardson (Elsevier, Amsterdam, 1996), p. 577-650.

${ }^{23}$ E. Bertel, Surf. Sci. 367, L61 (1996).

${ }^{24}$ R. C. Longo, V. S. Stepanyuk, and J. Kirschner, J. Phys.: Condens. Matter 18, 9143 (2006).

${ }^{25}$ M. W. Holmes, D. A. King, and J. E. Inglesfield, Phys. Rev. Lett. 42, 394 (1979).

${ }^{26}$ M. Bode, S. Krause, L. Berbil-Bautista, S. Heinze, and R. Wiesendanger, Surf. Sci. 601, 3308 (2007).

${ }^{27}$ F. Schiller, R. Keyling, E. V. Chulkov, and J. E. Ortega, Phys. Rev. Lett. 95, 126402 (2005).

${ }^{28}$ J. Schäfer, M. Hoinkis, D. Schrupp, E. Rothenberg, P. Blaha, and R. Claessen, Surf. Sci. 600, 3912 (2006). 